\title{
Frequency Dependence of Magnetopolarizability of Mesoscopic Grains
}

\author{
Ya. M. Blanter ${ }^{a}$ and A. D. Mirlin ${ }^{b, c, *}$ \\ ${ }^{a}$ Département de Physique Théorique, Université de Genève, CH-1211 Genève 4, Switzerland \\ ${ }^{b}$ Institut für Nanotechnologie, Forschungszentrum Karlsruhe, 76021 Karlsruhe, Germany \\ ${ }^{c}$ Institut für Theorie der Kondensierten Materie, Universität Karlsruhe, 76128 Karlsruhe, Germany
}

(November 4, 2018)

We calculate average magnetopolarizability of an isolated metallic sample at frequency $\omega$ comparable to the mean level spacing $\Delta$. The frequency dependence of the magnetopolarizability is described by a universal function of $\omega / \Delta$.

PACS numbers:73.20.Fz,73.23.-b,73.61.-r

Mesoscopic effects in electric polarizability of small metallic particles have been discussed in the literature starting from the seminal work by Gor'kov and Eliashberg (GE) [1]. The role of screening effects, which were not taken into account in the original paper [1], has been subsequently emphasized [2]. Whereas GE predicted a giant effect of level correlations on the polarizability, screening actually reduces this quantum effect to a relatively small correction to the classical value. Like other mesoscopic effects related to quantum interference, this correction is affected by magnetic field. It can thus be observed in magnetopolarizability of a grain, similarly to the weak localization correction to resistivity [3 5]. An experimental study of quantum corrections in electromagnetic response of an ensemble of $10^{5}$ mesoscopic samples has been reported recently [6, 7].

The aim of this paper is to calculate the dependence of the magnetopolarizability on the frequency $\omega$ of the applied electric field in the range $\omega \sim \Delta$, where $\Delta$ is the mean level spacing. This dependence is given by a universal function of the parameter $\omega / \Delta$. Note that the frequency in the experiments [6.7] was comparable to $\Delta$, and thus experimental verification of this scaling form is in principle feasible.

Previously we have demonstrated [8,5 how the supersymmetric sigma-model can be employed to calculate magnetopolarizability in the grand canonical ensemble. However, the experimentally relevant situation of isolated grains is appropriately described in the framework of the canonical ensemble. The crucial role of the statistical ensemble for the problem considered has been emphasized in Ref. 9] (see also Ref. [10]). Technically, calculation of the dynamical response within the canonical ensemble is more complicated, since it involves three-level correlation functions, which poses a serious obstacle to the application of the supersymmetry technique. Below, we bypass this difficulty by combining information on statistical properties of wavefunctions, obtained with the supersymmetry technique, with the three-level correlation function known from the random matrix theory (RMT).
The (complex-valued) polarizability is defined as $\boldsymbol{d}(\omega)=\alpha(\omega) \boldsymbol{E}(\omega)$, where $\boldsymbol{E}$ and $\boldsymbol{d}$ are the external electric field and the induced dipole moment, respectively. The expression for the ensemble-averaged magnetopolarizability $\alpha_{B}(\omega) \equiv \alpha(\omega, B)-\alpha(\omega, 0)$, where $B$ is an applied magnetic field (strong enough to break the timereversal symmetry) has the form [3,5]

$$
\alpha_{B}(\omega)=\frac{2 e^{2}}{E^{2}} \int d \boldsymbol{r}_{\mathbf{1}} d \boldsymbol{r}_{\mathbf{2}} \Phi\left(\boldsymbol{r}_{\mathbf{1}}\right) \delta \Pi\left(\boldsymbol{r}_{\mathbf{1}}, \boldsymbol{r}_{\mathbf{2}} ; \omega\right) \Phi\left(\boldsymbol{r}_{\mathbf{2}}\right),
$$

where $\Phi(\boldsymbol{r})$ is the local potential in the sample (resulting from screening of the external electric field), the symbol $\boldsymbol{\delta}$ denotes the difference between quantities with and without magnetic field, and $\Pi$ is the polarization operator,

$$
\begin{aligned}
\Pi\left(\boldsymbol{r}_{\mathbf{1}}, \boldsymbol{r}_{\mathbf{2}} ; \omega\right) & =\left\langle\sum_{m \neq n} \psi_{m}^{*}\left(\boldsymbol{r}_{\mathbf{1}}\right) \psi_{n}\left(\boldsymbol{r}_{\mathbf{1}}\right) \psi_{n}^{*}\left(\boldsymbol{r}_{\mathbf{2}}\right) \psi_{m}\left(\boldsymbol{r}_{\mathbf{2}}\right)\right. \\
& \left.\times \frac{n_{F}\left(\epsilon_{m}\right)-n_{F}\left(\epsilon_{n}\right)}{\omega-\epsilon_{m}+\epsilon_{n}}\right\rangle .
\end{aligned}
$$

Here $m$ and $n$ label exact single-particle states, the angular brackets denote the ensemble averaging, and it is assumed that $\omega$ has an (infinitesimally small) positive imaginary part, $\omega \equiv \omega+i 0$. We will consider the lowtemperature limit, $T \ll \Delta$, thus setting $T=0$ in the sequel. Substitution of (2) into (11) yields

$$
\begin{aligned}
\alpha_{B}(\omega) & =\frac{2 e^{2}}{E^{2}} \delta\left\langle\sum_{\epsilon_{n}<\epsilon_{F}<\epsilon_{m}}\left|\Phi_{m n}\right|^{2}\right. \\
& \left.\times\left(\frac{1}{\epsilon_{m}-\epsilon_{n}-\omega}+\frac{1}{\epsilon_{m}-\epsilon_{n}+\omega}\right)\right\rangle,
\end{aligned}
$$

where $\Phi_{m n}=\int d \boldsymbol{r} \psi_{m}^{*}(\boldsymbol{r}) \Phi(\boldsymbol{r}) \psi_{n}(\boldsymbol{r})$.

The canonical ensemble is realized by pinning the Fermi-level to one of the single-particle levels $\epsilon_{k}: \epsilon_{F}=$ $\epsilon_{k}+0$. Splitting the sum in (3) into two contributions with $n=k$ and $n \neq k$, we find

$$
\begin{aligned}
\alpha_{B} & =\frac{2 e^{2}}{E^{2} \Delta^{2}} \delta\left\{\int_{+0}^{\infty} d \epsilon\left(\frac{1}{\epsilon-\omega}+\frac{1}{\epsilon+\omega}\right)\right. \\
& \left.\times\left[R_{2}(\epsilon) \Delta+\int_{+0}^{\epsilon-0} d \epsilon_{1} R_{3}\left(\epsilon, \epsilon_{1}\right)\right]\left\langle|\Phi|^{2}\right\rangle_{\epsilon}\right\},
\end{aligned}
$$

where $R_{2}(\epsilon)$ and $R_{3}\left(\epsilon, \epsilon_{1}\right)$ are the two-level and the threelevel correlation functions (normalized to unity at $\epsilon, \epsilon_{1} \gg$ $\Delta)$, 


$$
\begin{aligned}
R_{2}(\epsilon) & =\Delta^{2}\left\langle\sum_{i j} \delta\left(E-E_{i}\right) \delta\left(E+\epsilon-E_{j}\right)\right\rangle ; \\
R_{3}\left(\epsilon, \epsilon_{1}\right) & =\Delta^{3}\left\langle\sum_{i j k} \delta\left(E-E_{i}\right) \delta\left(E+\epsilon-E_{j}\right)\right. \\
& \left.\times \delta\left(E+\epsilon_{1}-E_{k}\right)\right\rangle,
\end{aligned}
$$

and $\left\langle|\Phi|^{2}\right\rangle_{\epsilon}$ is the average squared matrix element,

$$
\begin{aligned}
\left\langle|\Phi|^{2}\right\rangle_{\epsilon} & =R_{2}^{-1}(\epsilon) \Delta^{2}\left\langle\sum_{i j}\left|\Phi_{i j}\right|^{2}\right. \\
& \left.\times \delta\left(E-E_{i}\right) \delta\left(E+\epsilon-E_{j}\right)\right\rangle .
\end{aligned}
$$

When writing Eq. (画), we decoupled the wave function correlations (6) from the three-level correlation function $R_{3}\left(\epsilon, \epsilon_{1}\right)$. Indeed, we know from the supersymmetry calculations of two-level correlation functions [11] that (i) the level correlation function has the RMT form, up to $1 / g^{2}$ corrections; (ii) the wavefunction correlations have no dependence on $\omega / \Delta$ in the order $1 / g$. Here $g \sim E_{c} / \Delta \gg 1$ is the dimensionless conductance of the grain, $E_{c}$ is the Thouless energy, and we consider the frequency range $\omega \ll E_{c}$. We make thus an (extremely plausible) assumption that these properties hold also for higher order correlation functions, which allows us to proceed in the case of the canonical ensemble.

We begin the evaluation of Eq. (4) by considering the term which contains the three-level correlator. We write $R_{3}\left(\epsilon, \epsilon_{1}\right)=R_{2}(\epsilon)+\tilde{R}_{3}\left(\epsilon, \epsilon_{1}\right)$ and denote the corresponding contributions as $\alpha_{B}^{(1)}$ and $\alpha_{B}^{(2)}$. In the leading order in $1 / g$ the $\epsilon$-integral in $\alpha_{B}^{(1)}$ is a sum of contributions from the regions $\epsilon \sim E_{c}$ and $\epsilon \sim \Delta$ (to be denoted as $\alpha_{B}^{(1 a)}$ and $\alpha_{B}^{(1 b)}$ respectively). In the former we can neglect $\omega$, which yields after taking into account the orthogonality and completeness of the eigenfunctions [ 4 汭],

$$
\begin{aligned}
\alpha_{B}^{(1 a)} & =\frac{4 e^{2}}{E^{2} \Delta^{2}} \boldsymbol{\delta} \int_{+0}^{\infty} d \epsilon R_{2}(\epsilon)\left\langle|\Phi|^{2}\right\rangle_{\epsilon} \\
& =-\frac{2 e^{2}}{E^{2} \Delta}\left\langle\left|\Phi_{m m}\right|^{2}\right\rangle=\frac{2 e^{2}}{E^{2} \Delta}\left\langle|\Phi|^{2}\right\rangle_{0},
\end{aligned}
$$

where we defined

$$
\begin{aligned}
\left\langle|\Phi|^{2}\right\rangle_{0} & =\left\langle|\Phi|^{2}\right\rangle_{\epsilon \ll E_{c}} \\
& =\frac{1}{V^{2}} \int d \boldsymbol{r}_{\mathbf{1}} d \boldsymbol{r}_{\mathbf{2}} \Phi\left(\boldsymbol{r}_{\mathbf{1}}\right) \Phi\left(\boldsymbol{r}_{\mathbf{2}}\right) \Pi_{D}\left(\boldsymbol{r}_{\mathbf{1}}, \boldsymbol{r}_{\mathbf{2}}\right),
\end{aligned}
$$

and $\Pi_{D}$ is the diffusion propagator satisfying

$$
-D \nabla^{2} \Pi_{D}\left(\boldsymbol{r}_{\mathbf{1}}, \boldsymbol{r}_{\mathbf{2}}\right)=(\pi \nu)^{-1}\left[\delta\left(\boldsymbol{r}_{\mathbf{1}}-\boldsymbol{r}_{\mathbf{2}}\right)-V^{-1}\right],
$$

with the boundary condition $\nabla_{\boldsymbol{n}} \Pi_{D}=0$. In the contribution $\alpha_{B}^{(1 b)}$ we can neglect the $\epsilon$-dependence of the matrix element, thus replacing it by $\left\langle|\Phi|^{2}\right\rangle_{0}$,

$$
\begin{aligned}
\alpha_{B}^{(1 b)} & =\frac{2 e^{2}}{E^{2} \Delta^{2}}\left\langle|\Phi|^{2}\right\rangle_{0} \\
& \times \int_{+0}^{\infty} d \epsilon\left(\frac{1}{\epsilon-\omega}+\frac{1}{\epsilon+\omega}\right) \epsilon \boldsymbol{\delta} R_{2}(\epsilon) .
\end{aligned}
$$

The same is valid for the term $\alpha_{B}^{(2)}$, as well as for the remaining contribution of the first term in square brackets in (何. Collecting everything, we finally get

$$
\alpha_{B}=\frac{2 e^{2}}{E^{2} \Delta}\left\langle|\Phi|^{2}\right\rangle_{0} F(\omega),
$$

where

$$
\begin{aligned}
F(\omega) & =1+\int_{+0}^{\infty} \frac{d \epsilon}{\Delta}\left(\frac{1}{\epsilon-\omega}+\frac{1}{\epsilon+\omega}\right) \\
& \times\left[\epsilon \boldsymbol{\delta} R_{2}(\epsilon)+\Delta \boldsymbol{\delta} R_{2}(\epsilon)+\int_{+0}^{\epsilon-0} d \epsilon_{1} \boldsymbol{\delta} \tilde{R}_{3}\left(\epsilon, \epsilon_{1}\right)\right]
\end{aligned}
$$

Since the integrals in (12) are determined by the range $\epsilon, \epsilon_{1} \sim \Delta$, we can use the RMT results 12 for the level correlation functions $R_{2}$ and $\tilde{R}_{3}$ entering this formula. Therefore, $F(\omega)$ is in fact a universal function of the dimensionless parameter $s=\omega / \Delta$. Real and imaginary parts of this function describe the influence of the magnetic field on polarizability and absorption of grains, respectively, and are plotted in Fig. 1.

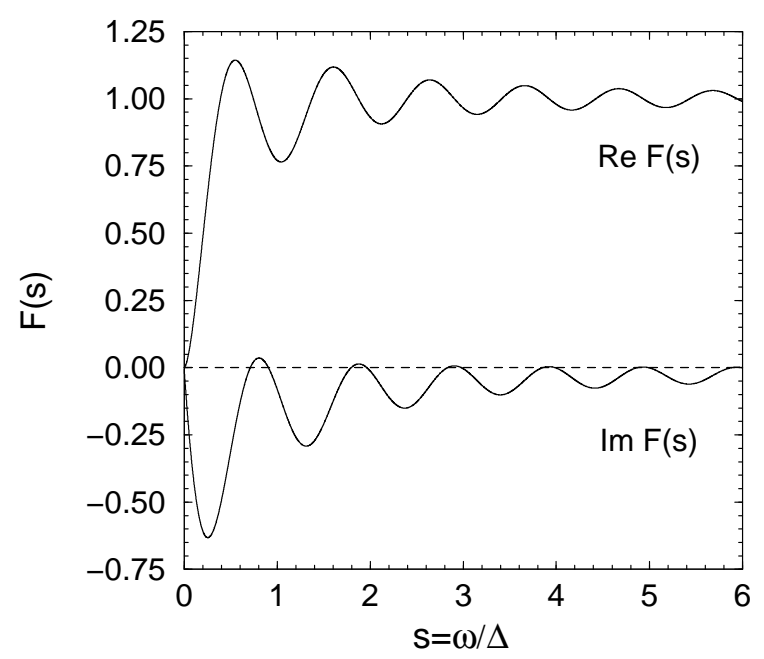

FIG. 1. Real and imaginary parts of the function $F$ [Eq. (12)] representing the frequency dependence of the magnetopolarizability $\alpha_{B}(\omega)$.

Note that $F(s)=0$ at $s=0$, which is an identity relating the two-level and the three-level correlation functions entering Eq. (12). This identity can be derived by using the invariance of the level correlations with respect to a perturbation 13.

As is seen from Fig. 1, our results predict a positive magnetopolarizability for all values of $\omega$ and a negative magnetoabsorption for almost all $\omega$. This is in qualitative agreement with the findings of Ref. [7], where the 
measurements have been performed at a frequency corresponding to $s \simeq 0.21$. It would be very interesting to have the experimental data for several frequencies in the range $\omega \sim \Delta$ in order to check our prediction for the universal scaling function $F(s)$ describing the frequency dependence of the magnetopolarizability induced by the level and eigenfunction statistics in the grains.

We are grateful to $\mathrm{H}$. Bouchiat for stimulating discussions. This work was supported by the Swiss National Science Foundation (Y. M. B.) and the SFB 195 der Deutschen Forschungsgemeinschaft (A. D. M.). We gratefully acknowledge the hospitality of the Lorentz Center in Leiden, where this work was completed.

* Also at St.Petersburg Nuclear Physics Institute, 188350 St.Petersburg, Russia.

[1] L. P. Gor'kov and G. M. Eliashberg, Zh. Eksp. Teor. Fiz. 48, 1407 (1965) [Sov. Phys. JETP 21, 940 (1965)].

[2] S. Strässler, M. J. Rice, and P. Wyder, Phys. Rev. B 6, 2575 (1972); M. J. Rice, W. R. Schneider, and S. Strässler, Phys. Rev. B 8, 474 (1973).

[3] K. B. Efetov, Phys. Rev. Lett. 76, 1908 (1996).

[4] Y. Noat, B. Reulet, and H. Bouchiat, Europhys. Lett. 36, 701 (1996).

[5] Ya. M. Blanter and A. D. Mirlin, Phys. Rev. B 57, 4566 (1998).

[6] Y. Noat, H. Bouchiat, B. Reulet, and D. Mailly, Phys. Rev. Lett. 80, 4955 (1998).

[7] R. Deblock, Y. Noat, H. Bouchiat, B. Reulet, and D. Mailly, Phys. Rev. Lett. 84, 5379 (2000).

[8] Ya. M. Blanter and A. D. Mirlin, Phys. Rev. B 53, 12601 (1996).

[9] B. I. Shklovskii, Pis'ma Zh. Eksp. Teor. Fiz. 36, 287 (1982) [JETP Lett. 36, 352 (1982)].

[10] A. Kamenev, B. Reulet, H. Bouchiat, and Y. Gefen, Europhys. Lett. 28, 391 (1994).

[11] For review, see K. B. Efetov, Supersymmetry in Disorder and Chaos (Cambridge University Press, New York, 1997); A. D. Mirlin, Phys. Rep. 326, 259 (2000).

[12] M. L. Mehta, Random Matrices, Academic Press Inc. (N. Y.) (1991); O. Bohigas, in: Chaos and Quantum Physics, Les Houches Session LII, 1989, Ed. by M.-J. Giannoni, A. Voros, and J. Zinn-Justin, Elsevier Science B. V. (N. Y.) (1991), p.87.

[13] In our paper [5] a non-zero value of $\alpha_{B}(0)$ was given, which was a result of an unfortunate arythmetic error in evaluation of the $\omega=0$ limit of the integral (12). We are grateful to $\mathrm{H}$. Bouchiat for giving a qualitative argument implying $\alpha_{B}(0)=0$, which helped us to find this error. 